\title{
Systematic Screening for Atrial Fibrillation in Patients at Moderate-to-High Risk of Stroke - Potential to Increase the Atrial Fibrillation Detection Rate (SCAN-AF) -
}

\author{
Eiichi Watanabe, MD, PhD; Naohiko Takahashi, MD, PhD; \\ Ronald Aronson, MD; Ako Ohsawa, BSc; Yuriko Ishibashi; \\ Yuji Murakawa, MD, PhD for the SCAN-AF Investigators
}

\begin{abstract}
Background: To determine the rate of undiagnosed atrial fibrillation (AF) we screened for AF using an oscillometric blood pressure (BP) monitor device followed by a single-lead handheld electrocardiogram (ECG), with confirmation by 12-lead ECG as the reference standard.

Methods and Results: From October 2017 to August 2019, 1,148 patients were enrolled without known AF, who were aged $\geq 65$ years with moderate-to-high stroke risk, at 71 centers in Japan. After exclusion of 7 patients with confirmed AF at the index visit, 1,141 patients were asked to use an oscillometric BP monitor twice daily for 2 weeks (max: 4 weeks) to detect an irregular pulse. The BP monitor detected an irregular pulse in 481 patients, of which 1 patient had confirmed AF. Thereafter, 480 patients were instructed to acquire ECGs twice daily for an additional 2 weeks (max: 4 weeks) using a single-lead handheld ECG device. The handheld ECG device detected irregular rhythm in 41 patients, of which 1 patient had confirmed AF. In total, undiagnosed AF was confirmed in $9(0.8 \%)$ patients of the overall study cohort during the 24-week follow-up period.
\end{abstract}

Conclusions: Sequential use of a BP monitor and handheld ECG for 4 weeks is a practical strategy for identifying undiagnosed AF in Japanese people at heightened risk of stroke.

Key Words: Arrhythmia; Atrial fibrillation; Diagnosis; Electrocardiography; Screening

A trial fibrillation (AF) is a treatable risk factor for stroke, and its prevalence is increasing. ${ }^{\mathbf{1} 2}$ In 2005, the number of patients with AF in Japan was 716,000 ; it is predicted to increase to 1.03 million, or $1.1 \%$ of the national population, by $2050 .{ }^{3}$ Because approximately one-half of patients with AF are asymptomatic or have non-specific symptoms, ${ }^{4}$ many are undiagnosed and may not receive an oral anticoagulant to reduce the risk of strokes. Therefore, screening for undiagnosed AF is becoming increasingly important. ${ }^{5-9}$ In addition to traditional methods, technologic advances have enabled a variety of approaches to facilitate AF screening. ${ }^{69}$ Newer, non-invasive devices include the oscillometric blood pressure (BP) monitor, which can detect an irregular pulse, the photoplethysmography-based pulse monitor, long-term electrocardiogram (ECG) patches, and single-lead handheld ECG.69 Of these, handheld ECG devices are appealing for AF screening because they are easy to handle, portable, use diagnostic algorithms to perform automated analyses of heart rhythms, and record and store ECG traces that cardiologists can later analyze. Nevertheless, single-lead handheld ECG devices still require 12-lead ECG recordings for confirmation of AF diagnosis. ${ }^{9}$ Care must be taken when screening for asymptomatic AF because there is a possibility for misinterpretation of ECGs by general practitioners, subsequent unnecessary treatment, increased healthcare resource use, and impaired quality of life for patients beyond symptom burden or

Received September 24, 2021; revised manuscript received December 16, 2021; accepted January 5, 2022; J-STAGE Advance Publication released online February 5, 2022 Time for primary review: 18 days

Division of Cardiology, Department of Internal Medicine, Fujita Health University Bantane Hospital, Nagoya (E.W.); Department of Cardiology and Clinical Examination, Oita University Faculty of Medicine, Oita (N.T.), Japan; Department of Cardiovascular Clinical Development, Bristol Myers Squibb, Lawrenceville, NJ (R.A.), USA; Pfizer Japan Inc, Tokyo (A.O.); Bristol Myers Squibb, Tokyo (Y.I.); and Fourth Department of Internal Medicine, Teikyo University, Tokyo (Y.M.), Japan

Y.I. is the Former employee.

Mailing address: Eiichi Watanabe, MD, PhD, Division of Cardiology, Department of Internal Medicine, Fujita Health University Bantane Hospital, Nagoya 454-0012, Japan. E-mail: enwatan@fujita-hu.ac.jp

All rights are reserved to the Japanese Circulation Society. For permissions, please e-mail: cj@j-circ.or.jp

ISSN-1346-9843 


\section{Table 1. Baseline Patient Characteristics}

\begin{tabular}{|c|c|}
\hline Characteristic & $\begin{array}{c}\text { All patients } \\
(n=1,148)\end{array}$ \\
\hline Age, years & $74.0(69.0,79.0)$ \\
\hline Age $\geq 75$ years & $568(49.5)$ \\
\hline \multicolumn{2}{|l|}{ Sex } \\
\hline Female & $568(49.5)$ \\
\hline Male & $580(50.5)$ \\
\hline Weight, kg & $59.7(52.1,68.0)$ \\
\hline $\mathrm{BMI}, \mathrm{kg} / \mathrm{m}^{2}$ & $24.0(21.9,26.4)$ \\
\hline Heart rate, beat $/ \mathrm{min}$ & $71.0(65.0,78.0)$ \\
\hline $\mathrm{SBP}, \mathrm{mmHg}$ & $137.0(127.0,150.0)$ \\
\hline $\mathrm{DBP}, \mathrm{mmHg}$ & $80.0(72.0,88.0)$ \\
\hline $\mathrm{CHADS}_{2}$ score & $2.0(1.0,2.0)$ \\
\hline $\mathrm{CHA}_{2} \mathrm{DS}_{2}-\mathrm{VASc}$ score & $4.0(3.0,4.0)$ \\
\hline \multicolumn{2}{|l|}{$\mathrm{CHA}_{2} \mathrm{DS}_{2}$-VASc score distribution } \\
\hline 2 & $178(15.5)$ \\
\hline 3 & 377 (32.8) \\
\hline 4 & $349(30.4)$ \\
\hline 5 & $165(14.3)$ \\
\hline 6 & $68(5.9)$ \\
\hline 7 & $10(0.9)$ \\
\hline 8 & $1(<0.1)$ \\
\hline \multicolumn{2}{|l|}{ Medical history } \\
\hline Hypertension & $1,062(92.5)$ \\
\hline Congestive heart failure & $51(4.4)$ \\
\hline Diabetes mellitus & $334(29.1)$ \\
\hline Stroke or transient ischemic attack & $25(2.2)$ \\
\hline Vascular disease & $213(18.6)$ \\
\hline Chronic kidney disease & $119(10.4)$ \\
\hline Chronic obstructive pulmonary disease & $26(2.3)$ \\
\hline Hepatic disease & $91(7.9)$ \\
\hline Sleep apnea syndrome & $22(1.9)$ \\
\hline \multicolumn{2}{|l|}{ Smoker } \\
\hline Current & $101(8.9)$ \\
\hline Former & $375(32.8)$ \\
\hline \multicolumn{2}{|l|}{ Alcohol history } \\
\hline Current & $517(45.0)$ \\
\hline Former & $110(9.6)$ \\
\hline \multicolumn{2}{|l|}{ Patient symptom questionnaire } \\
\hline History of palpitations & $128(11.1)$ \\
\hline History of faintness or syncope & $33(2.9)$ \\
\hline Anticoagulants & $25(2.2)$ \\
\hline
\end{tabular}

Data are presented as number (\%) or median (Q1, Q3) unless otherwise stated. BMI, body mass index; $\mathrm{CHADS}_{2}$, congestive heart failure, hypertension, age $\geq 75$ years, diabetes mellitus, stroke or transient ischemic attack; $\mathrm{CHA}_{2} \mathrm{DS}_{2}$-VASc, congestive heart failure, hypertension, age $\geq 75$ years, diabetes mellitus, stroke or transient ischemic attack, vascular disease, age 65-74 years, sex category; DBP, diastolic blood pressure; SBP, systolic blood pressure.

\section{disease severity. ${ }^{9}$}

To date, no trials have assessed whether treatment of screen-detected asymptomatic AF in older adults results in better health outcomes than treatment after detection via usual care or after symptoms develop. ${ }^{10}$

The purpose of the SCAN-AF study was to evaluate the detection rate of $\mathrm{AF}$ in patients without known $\mathrm{AF}$, who were aged $\geq 65$ years and at moderate-to-high risk of stroke. We applied a sequential use of oscillometric BP monitoring followed by a single-lead handheld ECG monitoring device, with confirmation by 12-lead ECG as the reference standard.

\section{Methods}

\section{Participants}

The SCAN-AF study was a multicenter, prospective, single-arm study conducted in 71 hospital-affiliated specialty outpatient sites in Japan between October 2017 and August 2019. Patients were included if they did not have known AF, were aged $\geq 65$ years and were at moderate-tohigh risk of stroke: $\mathrm{CHA}_{2} \mathrm{DS}_{2}-\mathrm{VASc}$ (congestive heart failure, hypertension, age $\geq 75$ years, diabetes, stroke, vascular disease, age 65-74 years, sex category) score $\geq 2$ or $\mathrm{CHADS}_{2}$ (congestive heart failure, hypertension, age $\geq 75$ years, diabetes, stroke) score $>1$. The exclusion criteria included previously documented AF, use of antiarrhythmic drugs, or inability to use the monitoring devices properly and/or comply with the required procedures.

\section{Study Procedures}

Patients who met the inclusion criteria had a 12-lead ECG at the index visit. Baseline symptoms were recorded by the study investigator. Patients without AF at the index visit were instructed to monitor their $\mathrm{BP}$ and pulse with the home oscillometric BP monitor (HEM-9200T; OMRON Healthcare, Kyoto, Japan) twice daily for 2 weeks (up to 4 weeks). The patients in whom an irregular pulse was detected underwent 12-lead ECGs at Visit 2. If the 12-lead ECG did not confirm AF, patients were asked to use a singlelead handheld ECG device (myBeat; UNION TOOL CO. Tokyo, Japan or HCG-801; OMRON Healthcare, Kyoto, Japan) to acquire an ECG $\geq 30$ s twice daily for an additional 2 weeks (up to 4 weeks). All patients had a 12-lead ECG at Visit 3, irrespective of the irregular rhythm detected by handheld ECG. From Visit 3 until the week 24 follow-up visit, investigators were allowed to use any device to detect AF if considered appropriate. All participants were followed for up to 24 weeks to assess adverse events. Two independent electrophysiologists adjudicated ECG tracings downloaded from 12-lead ECGs and handheld ECGs. If there was no consensus, the rhythm diagnosis was decided by discussion.

Two different definitions of a positive screen for AF were used in this study. "Confirmed AF" was defined as AF diagnosed by 12-lead ECG, and "probable AF" was defined as AF detected by handheld ECGs. At each scheduled visit, study personnel assessed the new diagnosis of $\mathrm{AF}$, ischemic or hemorrhagic stroke, transient ischemic attack, systemic embolism, major bleeding, vital status, and hospitalization from any cause.

\section{Endpoints}

The primary endpoint was the detection rate of all (prevalent and paroxysmal) AF confirmed by 12-lead ECG during the 24-week study period.

\section{Ethics}

This study was conducted in accordance with the International Society for Pharmacoepidemiology Guidelines for Good Pharmacoepidemiology Practices and applicable regulatory requirements, and in accordance with the ethical principles of the Declaration of Helsinki. All patients 


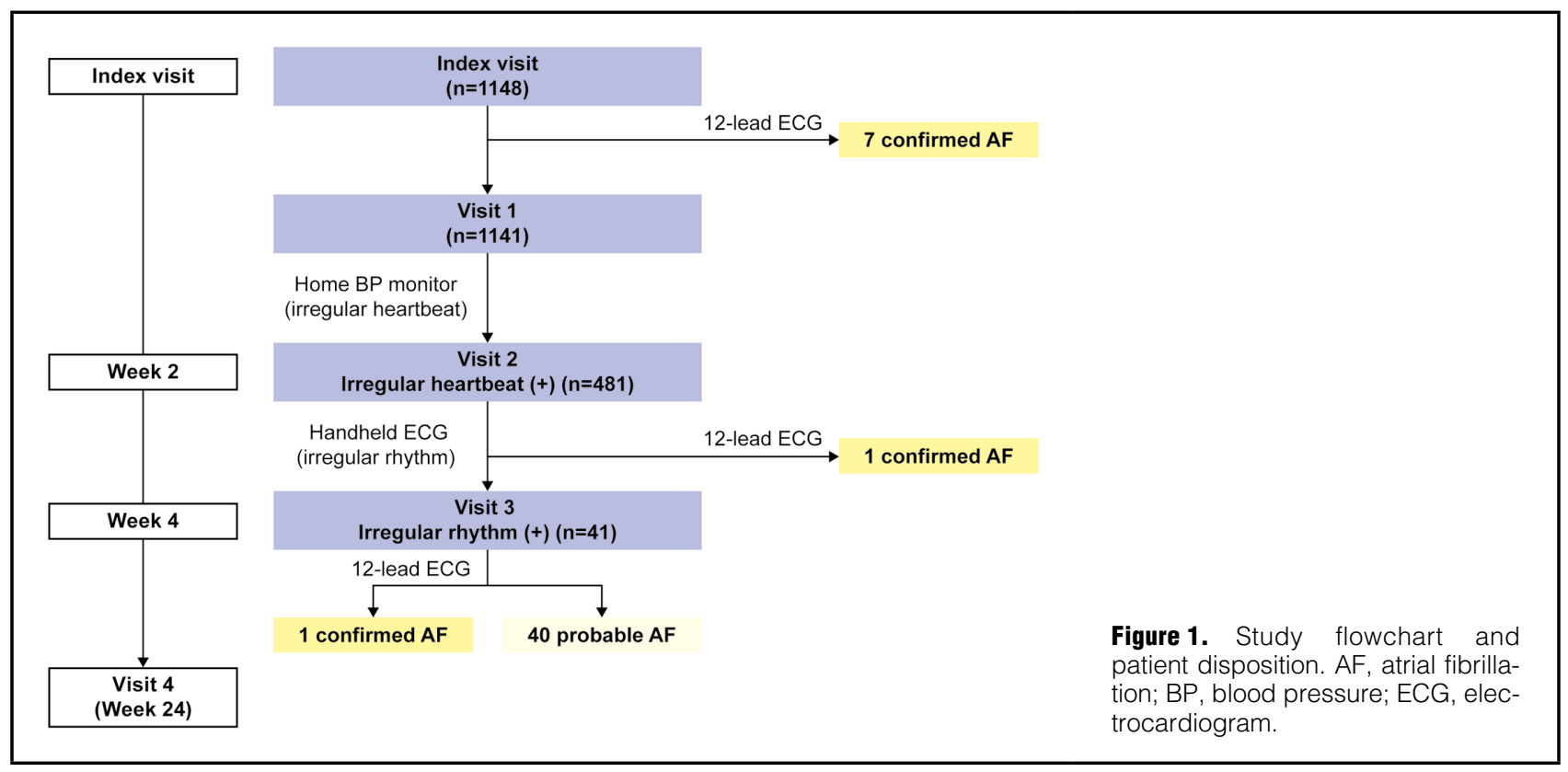

\begin{tabular}{|lcc|}
\hline \multicolumn{2}{|c|}{ Table 2. Detection Rate of Prevalence of All Irregular Pulse and Previously Undiagnosed AF } \\
& Number of patients & $\begin{array}{c}\text { Detection rate, \% } \\
\text { (95\% } \mathbf{~ C l )}\end{array}$ \\
Total number of patients & 1,148 & \\
Irregular pulse detected by home BP monitoring & 481 & $41.9(39.0-44.7)$ \\
Probable AF & 41 & $3.57(2.50-4.64)$ \\
Confirmed AF & 9 & $0.78(0.27-1.29)$ \\
\hline
\end{tabular}

The $95 \%$ Cls are based on the Wald asymptotic confidence limits. Confirmed AF indicates AF confirmed by 12-lead $\mathrm{ECG}$, and probable AF indicates AF detected by handheld single-lead ECG. AF, atrial fibrillation; BP, blood pressure; $\mathrm{Cl}$, confidence interval; ECG, electrocardiogram.

provided written informed consent. The study was registered with the University Hospital Medical Information Network Clinical Trial Registry, number UMIN000029473. The protocol, amendments, and patient-informed consent received appropriate approval by the IRB/Independent Ethics Committee or other applicable review board as required by local law prior to initiation of study at the site. The list of site principal investigators is available in the Supplementary Appendix.

\section{Statistical Analysis}

A sample of 1,200 patients allowed for the calculation of a $95 \%$ confidence interval $(\mathrm{CI})$ of $2-4 \%$ for the primary endpoint, assuming a $3 \%$ rate of detection of undiagnosed AF. Frequency distribution and summary statistics (median [Q1, Q3]) are presented for baseline demographics and clinical characteristics. For the primary endpoint, descriptive statistics are provided, including the detection rate and its $95 \%$ CI based on the Wald asymptotic confidence limits. Sensitivity and specificity for AF diagnosis were calculated as simple proportions with corresponding Clopper-Pearson exact CI for the handheld ECG device for AF detection. All analyses were performed using an intention-to-screen principle of all patients, regardless of device use, adherence, or duration of participation. Statistical analyses were performed with $\mathrm{R}$ project software $(\mathrm{R}$ foundation, Vienna, Austria).

\section{Results}

A total of 1,148 patients were included in the study. The median age at baseline was 74.0 years and $50.5 \%$ were male (Table 1). The median $\mathrm{CHADS}_{2}$ score was 2.0 and the median $\mathrm{CHA}_{2} \mathrm{DS}_{2}-\mathrm{VASc}$ score was 4.0. Figure 1 shows the study flow and results. At the index visit, 7 patients were confirmed to have AF by 12-lead ECG. The oscillometric BP monitoring device detected an irregular pulse in $481 / 1,141$ patients $(42.2 \%)$, of which only 1 had confirmed AF. Of the 480 patients who used the handheld ECGs (myBeat: $n=184$; HCG-801: $n=299$ [3 patients used both devices]), 41 patients had probable AF and AF was confirmed by 12 -lead ECG in 1 patient. In total, $9(0.8 \%)$ patients had confirmed AF and 41 patients had probable AF detected by handheld ECG (Table 2). The time course of probable AF detection is shown in Figure 2. Of the 41 patients, $28(68.3 \%)$ were detected within the first week of monitoring and $39(95.1 \%)$ were detected within 2 weeks. Although the protocol specified that the handheld ECG should be used for 2 weeks, due to a protocol deviation, some patients used it for up to 25 days; 1 patient had probable AF after completion of 2 weeks of follow-up. The number of patients with an irregular pulse detected by the 


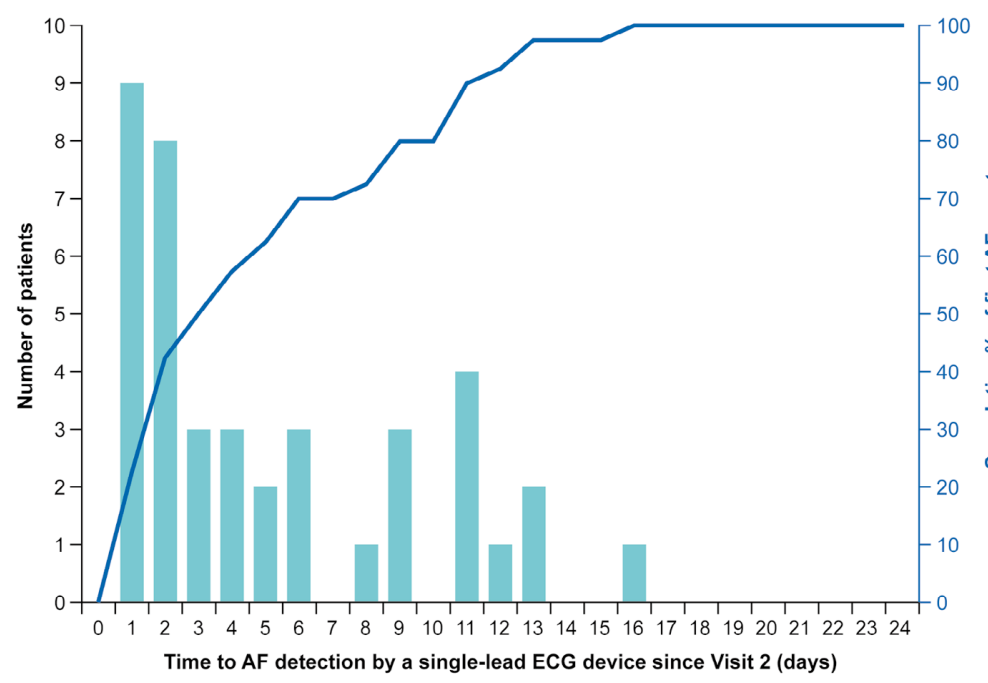

Figure 2. Time course of irregular rhythm detection by single-lead handheld ECG monitoring. Forty-one patients had irregular rhythm detected by single-lead handheld ECG monitoring. The bars show when irregular rhythm was first detected in days. The red line shows the cumulative incidence of first irregular rhythm. AF, atrial fibrillation; ECG, electrocardiogram.

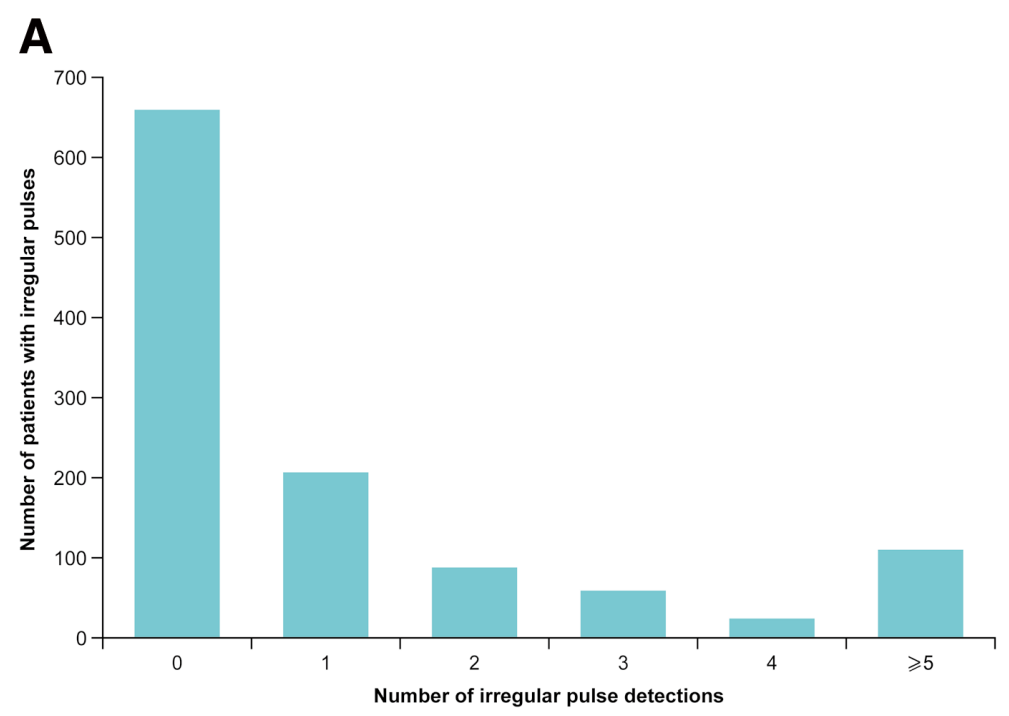

B

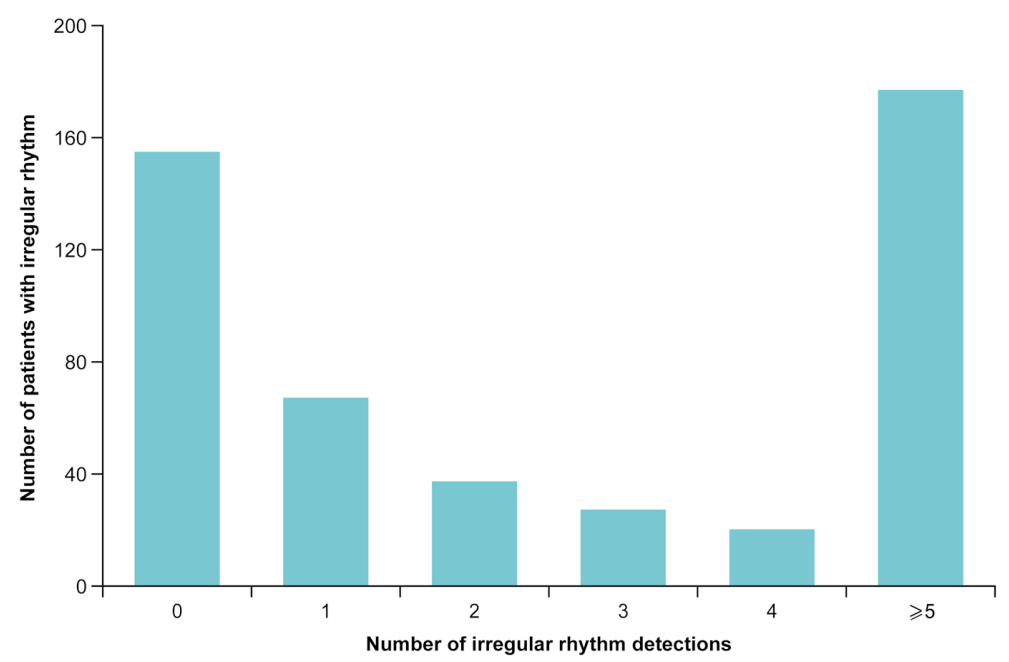

Figure 3. Number of irregular pulse and irregular rhythm detections. (A) Number of irregular pulses detected by the home BP monitor, and (B) number of irregular rhythms detected by the single-lead handheld ECG. $\mathrm{BP}$, blood pressure; ECG, electrocardiogram. 
BP monitor and probable AF detected by the handheld ECG are shown in Figure 3. The number of times AF was detected can be taken as a rough estimate of AF burden, with more detections reflecting a higher AF burden. Irregular pulses were recorded more than 5 times in $109(22.7 \%)$ patients, and 177 (36.8\%) patients had probable AF more than 5 times. Two patients with confirmed AF had both $\geq 5$ irregular pulse recordings and $\geq 5$ probable AF episodes. The data logs of the handheld ECGs showed that only 5/184 (2.7\%) patients using myBeat and 27/299 (9.0\%) patients using HCG-801 completed the scheduled 28 recordings for 2 weeks.

\section{Clinical Outcomes}

Follow up was completed for $95.6 \%$ of the patients at 24 weeks. Eight patients experienced 8 adverse events, of which 3 were serious ( 1 cerebral hemorrhage; 2 extended hospital stays for subarachnoid hemorrhage and acute myocardial infarction). During the study period, no patients died.

\section{Discussion}

SCAN-AF investigated the prevalence of undiagnosed AF in Japanese patients aged $\geq 65$ years with moderate-to-high risk of stroke detected with an oscillometric BP monitoring followed by a handheld single-lead ECG device with confirmation by 12-lead ECG as the reference standard. The handheld ECG device detected irregular rhythms (probable AF) in $41(3.6 \%)$ patients, and AF was confirmed by 12-lead ECG in $9(0.8 \%)$ patients. Although discontinuation rates for both devices were high, sequential use of a home BP monitor and single-lead handheld ECG for 4 weeks was a practical strategy for identifying undiagnosed AF.

Many studies have investigated the feasibility of population-based AF screening using a variety of screening methodologies. ${ }^{11-13}$ The yield of screening for identifying undiagnosed $\mathrm{AF}$ has ranged from approximately $0.1 \%$ to $5 \%{ }^{11}$ A retrospective cohort study in Japanese patients showed that the number of new AF cases detected through screening was greater in men than women, and that $80 \%$ of new AF cases in patients aged $\geq 65$ years had a sufficient stroke risk to receive a recommendation for oral anticoagulant treatment. ${ }^{14} \mathrm{~A}$ recent systematic review of $19 \mathrm{AF}$ screening studies showed that the age- and sex-adjusted detection rates for undiagnosed $\mathrm{AF}$ were $1.44 \%(95 \%$ $\mathrm{CI}=1.13-1.82 \%)$ and $0.41 \%(95 \% \mathrm{CI}=0.31-0.53 \%)$ for those aged $\geq 65$ years and $<65$ years, respectively. ${ }^{12}$ The detection rate for new AF increased progressively with age. ${ }^{12} \mathrm{AF}$ was detected by 12-lead ECG in 9 of the 1,148 screened patients, resulting in a detection rate of $0.78 \%$. Considering both confirmed AF (12-lead ECG) and probable $\mathrm{AF}$ ( $30 \mathrm{~s}$ of handheld ECG device recording), the number of patients with undiagnosed $\mathrm{AF}$ increases to 49/1,148 (4.27\%). A single 12-lead ECG might miss infrequent or short-lasting paroxysmal AF.

A unique strength of this study is that we first screened patients with a 12-lead ECG and then used a BP monitor, which is widely used in Japan, to further identify patients with undiagnosed AF. Shirasaki et al reported that $79 \%$ of the 11.2 million home electronic BP monitors sold worldwide have been sold in Japan. ${ }^{15}$ These devices can detect asymptomatic pulse irregularities consistent with atrial arrhythmias, including AF. ${ }^{16}$ An oscillometric BP monitor (OMRON 712C) has previously been shown to have high sensitivity (100\%), specificity (91\%), and accuracy (92\%) for the detection of AF. ${ }^{17}$ In this study, we did not calculate sensitivity or specificity of the BP monitor for detection of AF because only patients with an irregular pulse underwent 12-lead ECG. A systematic review and metaanalysis of 21 studies investigating the detection of irregular pulses caused by AF found that oscillometric BP monitors and non-12-lead ECG devices had the greatest diagnostic accuracy, and pulse palpitation had the lowest diagnostic accuracy. ${ }^{18}$

We diagnosed AF separately as either "confirmed AF" diagnosed with a 12-lead ECG or "probable AF" diagnosed with a handheld ECG. "Probable AF" describes single-lead recordings obtained with the handheld ECG that were consistent with AF based on the pattern of irregularity of the rhythm. A 12-lead ECG would be required to confirm AF with certainty.

Recent studies have reported successful identification of undiagnosed AF using smartwatches, but there are some limitations. ${ }^{19,20}$ A meta-analysis of 10 studies found that smartwatches had a high sensitivity and specificity, but the number of false positives in the asymptomatic population was higher than the number of true positive results. ${ }^{20}$ Realtime access to ECGs, however, may allow patients to play a more active role in AF screening. In the future, not only may improved artificial intelligence algorithms reduce the number of false positives, but they may also lead to a significant increase in automated diagnostics. ${ }^{21}$

This study used handheld ECG devices because they are easy for elderly people to use. In addition, the userfriendly, continuous patch ECG records may be suitable for the elderly population. ${ }^{8}$ Evidence regarding the optimal type of screening (systematic, opportunistic, or usual care) is limited. Two studies, both in patients aged $>65$ years, have shown similar rates of AF detection between opportunistic and systematic screening. ${ }^{22,23}$ The European Society of Cardiology (ESC) 2020 guidelines recommend opportunistic screening for AF by taking pulse or using ECG rhythm strips in patients aged $\geq 65$ years (Class I), and that systematic ECG screening should be considered to detect AF in individuals aged $\geq 75$ years or those at high risk of stroke (Class IIa). ${ }^{9}$ The National Heart Foundation of Australia and New Zealand (2018) strongly recommends opportunistic point-of-care screening in the clinic or community for individuals aged $\geq 65$ years by pulse palpation followed by ECG or handheld ECG. ${ }^{24}$

Whether widespread AF screening is both cost-effective and provides a net clinical benefit in people with undiagnosed AF is not established. A cluster randomized controlled trial found that both systematic screening and opportunistic screening in people aged $>65$ years identified more new cases of AF than routine practice; ${ }^{22}$ however, the cost of systematic screening was significantly greater than that of opportunistic screening from the perspective of the health service provider. This is supported by 2 other studies that have also shown systematic screening is associated with higher costs. ${ }^{22,25}$ In addition, the 2018 European Heart Rhythm Association, the Heart Rhythm Society, the Asian Pacific Heart Rhythm Society and the Sociedad Latino Americana de Estimulacion Cardiaca y Electrofisiologia (EHRA/HRS/APHRS/SOLAECE) guidelines state that systematic screening for AF is associated with higher costs than opportunistic screening. ${ }^{25}$ If opportunistic screening for $\mathrm{AF}$ became as commonplace among primary care providers as screening for hypertension, it might increase the 
rate of $\mathrm{AF}$ detection and subsequent treatment, thereby reducing the rate and costs of stroke in the older at-risk population.

Most previous AF screening studies in asymptomatic individuals have been observational studies that lack a control group, and therefore it is not possible to assess the balance of benefits and harms of AF screening. Several recent randomized trials provide evidence to support AF screening in asymptomatic older people,.$^{\mathbf{7 8 , 2 6}}$ Of these, the SCREEN-AF trial, conducted in individuals aged $\geq 75$ years with hypertension but without known AF, showed that the use of a wearable continuous ECG monitor increased AF detection 10-fold compared with standard care $(5.3 \%$ vs. $0.5 \%) .{ }^{8}$ Anticoagulation was initiated in $75 \%$ of patients who had AF detected in the screening group. ${ }^{8}$ The results of this study suggest the need for future research to determine the effect that screening will have on clinical outcomes like stroke, bleeding and death rates. One screening study for AF, STROKESTOP, has reported clinical outcomes in elderly patients in Sweden (5-year data for STROKESTOP II are expected in 2024). ${ }^{27}$ The primary outcome was the combined endpoint of ischemic stroke, systemic embolism, death, and major bleeding; patients who were screened had significantly fewer events than those in the overall population. ${ }^{27}$

Care should be taken when initiating screening programs, as abnormal results may cause anxiety for patients, ECG misinterpretation may lead to overdiagnosis and overtreatment, and ECGs may detect other abnormalities (true or false positives) that may lead to invasive tests and treatments that have the potential for serious harm..$^{10}$ Further study is needed to determine which patients would benefit most from AF screening and the best type of screening to be used.

\section{Study Limitations}

The screening methods used in this study have some intrinsic limitations, in that they rely on intermittent detection of the pattern of irregular pulses or rhythms recorded for only 30 s by a single-lead ECG, a technique that is consistent with, but not necessarily diagnostic for, AF. To confirm the diagnosis, a 12-lead ECG or a longer continuous recording with a wearable device is required. This study did not have a control arm to assess the rate of detection of undiagnosed AF in clinical practice. The rate of clinical events was low during the 24-week follow-up period, so the potential benefits and harms of screening for AF could not be assessed. The rate of AF detection may have been underestimated due to suboptimal sensitivity, improper use of the mobile devices, obtaining recordings only twice daily, and/or non-compliance. AF could have been underdetected by intermittent handheld ECG recordings if they were not taken overnight or at dawn. In addition, this study was designed to determine the incidence of undiagnosed AF, so we did not obtain the data needed to assess factors associated with development of AF, including sick sinus syndrome.

\section{Conclusions}

The sequential use of the home oscillometric BP monitor followed by a handheld single-lead ECG device for monitoring is a practical strategy for the identification of undiagnosed AF in Japanese people with moderate-to-high risk of stroke. Detection of underdiagnosed AF provides physi- cians with an option to consider anticoagulation therapy to reduce the risk of stroke in patients who would otherwise not have been offered this remarkably effective preventative therapy.

\section{Acknowledgments}

We thank Drs. Mari Amino (Tokai University School of Medicine), Masako Asakawa (JR Tokyo General Hospital), and Shinichi Niwano (Kitasato University School of Medicine) for diagnosing ECG tracings. Writing and editorial support was provided by Claire Line, PhD, at Caudex, and was funded by Pfizer and Bristol Myers Squibb.

\section{Sources of Funding}

This study was funded by Bristol Myers Squibb and Pfizer.

\section{Disclosures}

E.W. received remuneration for lecture fees from Daiichi-Sankyo, N.T. received remuneration for lecture fees from Daiichi Sankyo, Bristol Myers Squibb, Pfizer, Bayer Yakuhin, Nippon Boehringer Ingelheim, and Toa Eiyo, and is a member of Circulation Journal's Editorial Team. R.A. is an employee of and stockholder in Bristol Myers Squibb; Y.I. is a former employee of Bristol Myers Squibb. A.O. is an employee of Pfizer. Y.M. has nothing to declare.

\section{IRB Information}

The Teikyo University Ethics Committee (Number: Teirin 17-198), Fujita Health University Bantane Hospital (approval number: HM18-233), and Oita University Hospital (approval number: 1318) approved this study.

\section{Data Availability}

All relevant data have been included in this manuscript. The Bristol Myers Squibb policy on data sharing may be found at: https://www. bms.com/researchers-and-partners/independent-research/data-sharingrequest-process.html.

\section{References}

1. Go AS, Hylek EM, Phillips KA, Chang Y, Henault LE, Selby JV, et al. Prevalence of diagnosed atrial fibrillation in adults: National implications for rhythm management and stroke prevention: The AnTicoagulation and Risk Factors in Atrial Fibrillation (ATRIA) Study. JAMA 2001; 285: 2370-2375.

2. Schnabel RB, Yin X, Gona P, Larson MG, Beiser AS, McManus $\mathrm{DD}$, et al. 50 year trends in atrial fibrillation prevalence, incidence, risk factors, and mortality in the Framingham Heart Study: A cohort study. Lancet 2015; 386: 154-162.

3. Inoue H, Fujiki A, Origasa H, Ogawa S, Okumura K, Kubota I, et al. Prevalence of atrial fibrillation in the general population of Japan: An analysis based on periodic health examination. Int $J$ Cardiol 2009; 137: 102-107.

4. Siontis KC, Gersh BJ, Killian JM, Noseworthy PA, McCabe P, Weston SA, et al. Typical, atypical, and asymptomatic presentations of new-onset atrial fibrillation in the community: Characteristics and prognostic implications. Heart Rhythm 2016; 13: $1418-1424$.

5. Engdahl J, Andersson L, Mirskaya M, Rosenqvist M. Stepwise screening of atrial fibrillation in a 75-year-old population: Implications for stroke prevention. Circulation 2013; 127: 930-937.

6. Freedman B, Camm J, Calkins H, Healey JS, Rosenqvist M, Wang J, et al. Screening for atrial fibrillation: A report of the AF-SCREEN International Collaboration. Circulation 2017; 135: $1851-1867$.

7. Steinhubl SR, Waalen J, Edwards AM, Ariniello LM, Mehta RR, Ebner GS, et al. Effect of a home-based wearable continuous ECG monitoring patch on detection of undiagnosed atrial fibrillation: The mSToPS randomized clinical trial. JAMA 2018; 320: $146-155$.

8. Gladstone DJ, Wachter R, Schmalstieg-Bahr K, Quinn FR, Hummers E, Ivers N, et al. Screening for atrial fibrillation in the older population: A randomized clinical trial. JAMA Cardiol 2021; 6: $558-567$.

9. Hindricks G, Potpara T, Dagres N, Arbelo E, Bax JJ, BlomstromLundqvist C, et al. 2020 ESC Guidelines for the diagnosis and 
management of atrial fibrillation developed in collaboration with the European Association for Cardio-Thoracic Surgery (EACTS): The Task Force for the diagnosis and management of atrial fibrillation of the European Society of Cardiology (ESC). Developed with the special contribution of the European Heart Rhythm Association (EHRA) of the ESC. Eur Heart J 2021; 42: 373-498.

10. Jonas DE, Kahwati LC, Yun JDY, Middleton JC, CokerSchwimmer M, Asher GN. Screening for atrial fibrillation with electrocardiography: Evidence report and systematic review for the US Preventive Services Task Force. JAMA 2018; 320: 485-498.

11. Khurshid S, Healey JS, McIntyre WF, Lubitz SA. Populationbased screening for atrial fibrillation. Circ Res 2020; 127: $143-$ 154.

12. Lowres N, Olivier J, Chao TF, Chen SA, Chen Y, Diederichsen $\mathrm{A}$, et al. Estimated stroke risk, yield, and number needed to screen for atrial fibrillation detected through single time screening: A multicountry patient-level meta-analysis of 141,220 screened individuals. PLoS Med 2019; 16: e1002903.

13. Gudmundsdottir KK, Fredriksson T, Svennberg E, Al-Khalili F, Friberg L, Habel H, et al. Performance of pulse palpation compared to one-lead ECG in atrial fibrillation screening. Clin Cardiol 2021; 44: 692-698.

14. Nagata Y, Yamagami T, Nutbeam D, Freedman B, Lowres N. Incremental yield of ECG screening repeated annually over 4 years in an adult Japanese population without prior atrial fibrillation: A retrospective cohort study. BMJ Open 2020; 10: e035650.

15. Shirasaki O, Terada H, Niwano K, Nakanishi T, Kanai M, Miyawaki Y, et al. The Japan Home-Health Apparatus Industrial Association: Investigation of home-use electronic sphygmomanometers. Blood Press Monit 2001; 6: 303-307.

16. Noseworthy PA, Kaufman ES, Chen LY, Chung MK, Elkind MSV, Joglar JA, et al. Subclinical and device-detected atrial fibrillation: Pondering the knowledge gap: A scientific statement from the American Heart Association. Circulation 2019; 140: e944-e963.

17. Wiesel J, Wiesel D, Suri R, Messineo FC. The use of a modified sphygmomanometer to detect atrial fibrillation in outpatients. Pacing Clin Electrophysiol 2004; 27: 639-643.

18. Taggar JS, Coleman T, Lewis S, Heneghan C, Jones M. Accuracy of methods for detecting an irregular pulse and suspected atrial fibrillation: A systematic review and meta-analysis. Eur J Prev Cardiol 2016; 23: 1330-1338.
19. O'Sullivan JW, Grigg S, Crawford W, Turakhia MP, Perez M, Ingelsson $\mathrm{E}$, et al. Accuracy of smartphone camera applications for detecting atrial fibrillation: A systematic review and metaanalysis. JAMA Netw Open 2020; 3: e202064.

20. Strik M, Ploux S, Ramirez FD, Abu-Alrub S, Jaîs P, Haïssaguerre M, et al. Smartwatch-based detection of cardiac arrhythmias: Beyond the differentiation between sinus rhythm and atrial fibrillation. Heart Rhythm 2021; 18: 1524-1532.

21. Halvaei H, Svennberg E, Sörnmo L, Stridh M. Identification of transient noise to reduce false detections in screening for atrial fibrillation. Front Physiol 2021; 12: 672875.

22. Moran PS, Teljeur C, Ryan M, Smith SM. Systematic screening for the detection of atrial fibrillation. Cochrane Database Syst Rev 2016; 2016: CD009586.

23. Fitzmaurice DA, Hobbs FD, Jowett S, Mant J, Murray ET, Holder R, et al. Screening versus routine practice in detection of atrial fibrillation in patients aged 65 or over: Cluster randomised controlled trial. BMJ 2007; 335: 383.

24. Brieger D, Amerena J, Attia J, Bajorek B, Chan KH, Connell C, et al. National Heart Foundation of Australia and the Cardiac Society of Australia and New Zealand: Australian Clinical Guidelines for the Diagnosis and Management of Atrial Fibrillation 2018. Heart Lung Circ 2018; 27: 1209-1266.

25. Gorenek B, Boriani G, Dan GA, Fauchier L, Fenelon G, Huang $\mathrm{H}$, et al. European Heart Rhythm Association (EHRA) position paper on arrhythmia management and device therapies in endocrine disorders, endorsed by Asia Pacific Heart Rhythm Society (APHRS) and Latin American Heart Rhythm Society (LAHRS). Europace 2018; 20: 895-896.

26. Svennberg E, Friberg L, Frykman V, Al-Khalili F, Engdahl J, Rosenqvist M. Clinical outcomes in systematic screening for atrial fibrillation (STROKESTOP): A multicentre, parallel group, unmasked, randomised controlled trial. Lancet 2021; 398: $1498-$ 1506.

27. Svennberg E. EHRA 2021: The STROKESTOP-Study: Benefits of systematic screening for atrial fibrillation. Radcliffe Cardiology https://www.radcliffecardiology.com/EHRA-2021-STROKESTOPStudy-Svennberg (accessed August 10, 2021).

\section{Supplementary Files}

Please find supplementary file(s);

http://dx.doi.org/10.1253/circj.CJ-21-0813 